\title{
Editorial: the ACPSEM diagnostic imaging TEAP project
}

\author{
John Cormack
}

Published online: 15 June 2011

(C) Australasian College of Physical Scientists and Engineers in Medicine 2011

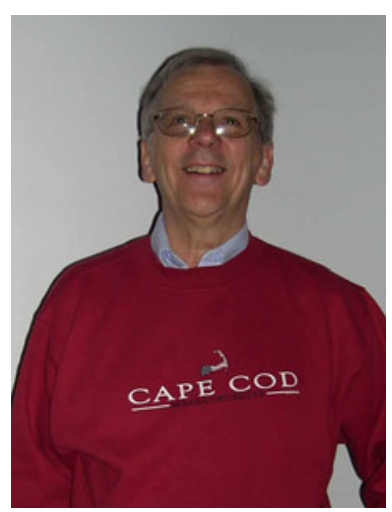

\section{Introduction}

Diagnostic imaging medical physicists (DIMPs)

The issue of names and acronyms for specialties and subspecialties of medical physics is largely political but very important-it helps define and differentiate us with government agencies, other professional organisations, regulators and even industrial bodies. In Australia, Radiation Oncology Medical Physicists have adopted the term "ROMPs" amongst themselves quite readily. With this in mind, after some debate with interested parties and consideration of all the options, the name Diagnostic Imaging Medical Physics (DIMP) was coined as a blanket term covering the interlinked sub-specialties of diagnostic radiology, nuclear medicine and hospital radiation safety. A

J. Cormack ( $\square)$

Australasian College of Physical Scientists and Engineers in Medicine, 247 Coward Street, Mascot, NSW 2020, Australia e-mail: jcormack@adam.com.au recent survey of college membership [1] has indicated that around $14 \%$ of members fall into the DIMP category (see Fig. 1).

Background and purpose of the project

Radiation dose from medical procedures has for the first time in history overtaken all other sources, both natural and man-made, as the most important contributor to exposure of humans, with the collective effective dose estimated to be 900,000 man Sieverts in the USA alone [2]. Diagnostic imaging is by far the largest contributing factor, with CT and molecular imaging alone contributing $75 \%$ of total medical radiation exposure [3]. As an indication of the magnitude of this dose, the total world population collective effective dose from the Chernobyl nuclear reactor accident accrued between 1986 and 2005 was around 300,000 man Sieverts [4]; in other words, the USA population receives a collective effective dose equivalent of about three Chernobyls every year! In Australia, because its smaller population, this equates to a Chernobyl every 5 years. It should be stressed that, for individual patients undergoing diagnostic imaging procedures, the benefits will generally far outweigh the risks; the above facts, however, do stress the extreme importance of monitoring, regulation and control in moderating population exposures.

The extent of the problem is such that The U.S. Food and Drug Administration (FDA) has announced an initiative to reduce unnecessary radiation exposure from CT, fluoroscopy, and nuclear medicine exams. The move is widely believed to be in response to reports of rising exposure to medical radiation in the US population. The US House of Representatives has held hearings on the issue of medical radiation. 


\section{ACPSEM Workforce Survey 2009}

ROMPS (55\%)

Nuclear Medicine (7\%)

Other (5\%)

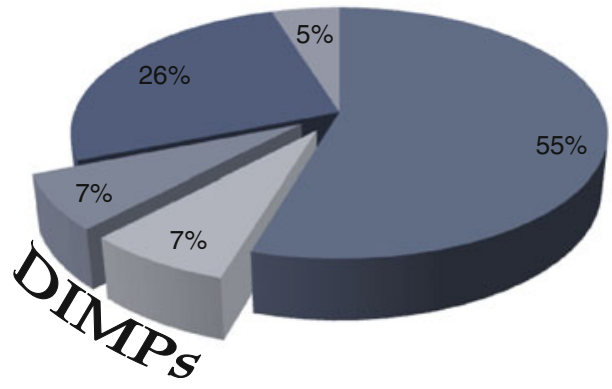

Fig. 1 ACPSEM workforce distribution, 2009. Total workforce is 605

The quality of medical images, and the diagnostic parameters derived from them, is also paramount in the medical management of patients. Incorrect diagnosis is the most important consequence of poor quality images. A poor image may cause the imaging physician to miss subtle, but diagnostically important, features (that is, it may reduce the sensitivity of the imaging procedure). Procedures which need to be repeated because of poor image quality, of course, also impose an additional risk to the patient. It is therefore crucial that adequate resources are provided for the monitoring of both radiation dose and image quality, and that this is carried out and controlled by competent professionals.

Medical physicists specialising in diagnostic imaging have the necessary training to provide the expertise required. Unfortunately, advancing clinical practice has placed additional demands on the services provided by diagnostic imaging medical physicists (DIMPs). This pressure has been compounded by the loss of experienced medical physicists due to natural attrition in an aging workforce, leading to a critical shortage of DIMPs not only in Australia, but worldwide.

Using the data obtained by Howell Round in his 2009 survey, plus data obtained from an additional survey and international benchmarks [5-7] to determine required/ actual staff ratios based on typical tasks performed by diagnostic imaging medical physicists, the ACPSEM has determined that the current workforce of experienced DIMPs needs to be increased by at least 40 over the next few years in order to meet demand. Failure to meet this target could have catastrophic consequences in terms of radiation risk and quality of diagnostic imaging services.

The purpose of this project was to develop ways of achieving the goal of an adequate and sustainable workforce and a Training, Education and Accreditation Programme (TEAP) which would meet current and future requirements.

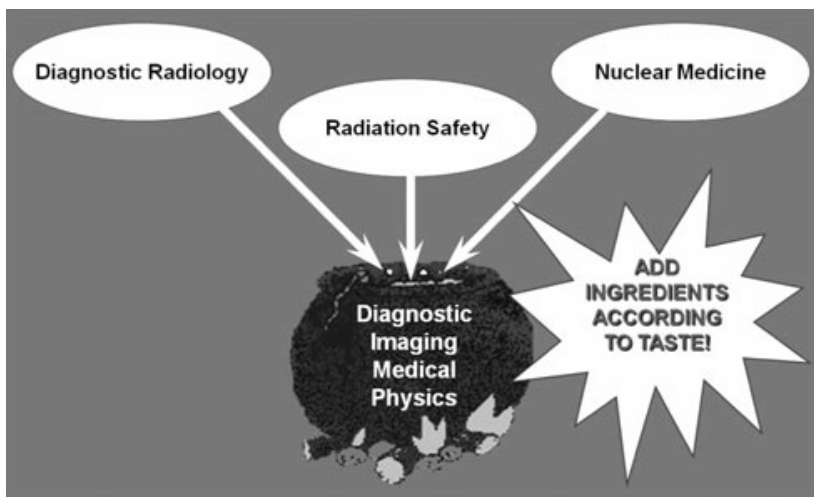

Fig. 2 Subspecialties incorporated in diagnostic imaging medical physics

\section{Current training programmes}

Diagnostic imaging medical physics comes in three distinct flavours (see Fig. 2); these are:

- Diagnostic radiology physics, in which medical imaging support is provided for imaging modalities such as radiography, mammography, CT, MRI and ultrasound.

- Nuclear medicine(or molecular imaging) physics, in which support is provided for gamma camera and PET imaging and other nuclear medicine procedures (including radionuclide therapy) which are normally carried out in a nuclear medicine facility.

- Radiation safety This is an important component of both of the above specialties. It involves patient and staff radiation monitoring, dosimetry and the implementation of procedures to ensure radiation safety and regulatory compliance on an institution-wide basis.

There are currently accreditation/training schemes in place for diagnostic radiology and nuclear medicine physics, but there is a considerable degree of overlap, particularly in the area of radiation safety.

Why a new training programme is needed

The demand for radiation-based imaging procedures has risen significantly in Australia over the past 10 years. This is partly due to the medical services required by an ageing population. The boundaries between the different subspecialties in medical imaging (ut supra) are also becoming more blurred; although a DIMP may be specialised on one area, it is increasingly common (and necessary) that some level of support in all of the specialties listed above is provided. In addition, there has been an expansion of the role of imaging in medical protocols, both for diagnosis and for intervention. An updated training programme is therefore required in order to accommodate these changes; in particular, it is imperative that an updated programme: 
- Addresses all of the core competencies for both the nuclear medicine and diagnostic radiology specialties.

- Identifies material overlapping both specialties for incorporation in common modules. As well as shortening the overall duration of accreditation, this would allow registrars undertaking accreditation in one specialty to have some experience of the elements of the other.

- Makes it possible to achieve accreditation in any one specialty in a reasonable time frame (e.g., 4 years maximum, including the M.Sc. course component).

- Offers dual accreditation in both specialities, with completion in a reasonable time frame (e.g., 5 years including the M.Sc. course). This will provide more flexibility for employers, and a broader career path for DIMP registrars.

- Is similar in generic structure to other TEAP programmes, including ROMPs,. In particular, there needs to be consistency in the evaluation methods used and grading levels for competencies.

- Incorporates ARPAB (Australasian Radiation Protection Accreditation Board) accreditation in ionising radiation safety at the radiation protection advisor/ professional health physicist level.

\section{The new DIMP TEAP}

\section{Introduction}

The new DIMP TEAP is strongly linked to the IAEA Clinical training programmes for nuclear medicine and diagnostic radiology. In order to address the current and ongoing needs of medical imaging facilities in Australia and New Zealand, however, modules from both programmes have been selected and adapted to provide a broader-based training covering all imaging modalities and radiation safety requirements. Accreditation with specialization in either diagnostic radiology or nuclear medicine is achieved in 4 years; dual accreditation can be achieved by undertaking some additional modules. In essence, registrars will complete all of the training modules related to their chosen specialty plus other modules related to achieving the core competencies required in a multi-modality medical imaging facility.

\section{Programme structure}

The ACPSEM DIMP training programme consists of four phases (see Fig. 3):

\section{Phase 1 (1 year)}

This is a didactic phase in which the registrar acquires the basic knowledge that is a pre-requisite for further clinical training. This will primarily be achieved through the completion of an ACPSEM accredited M.Sc. course.

Phase 2 (2 years)

Successful completion of Phase 1 is a pre-requisite for Phase 2. This phase is common to all the diagnostic imaging specialties. During this period the registrar will complete training modules which will ensure basic
Fig. 3 Structure of the new ACPSEM TEAP

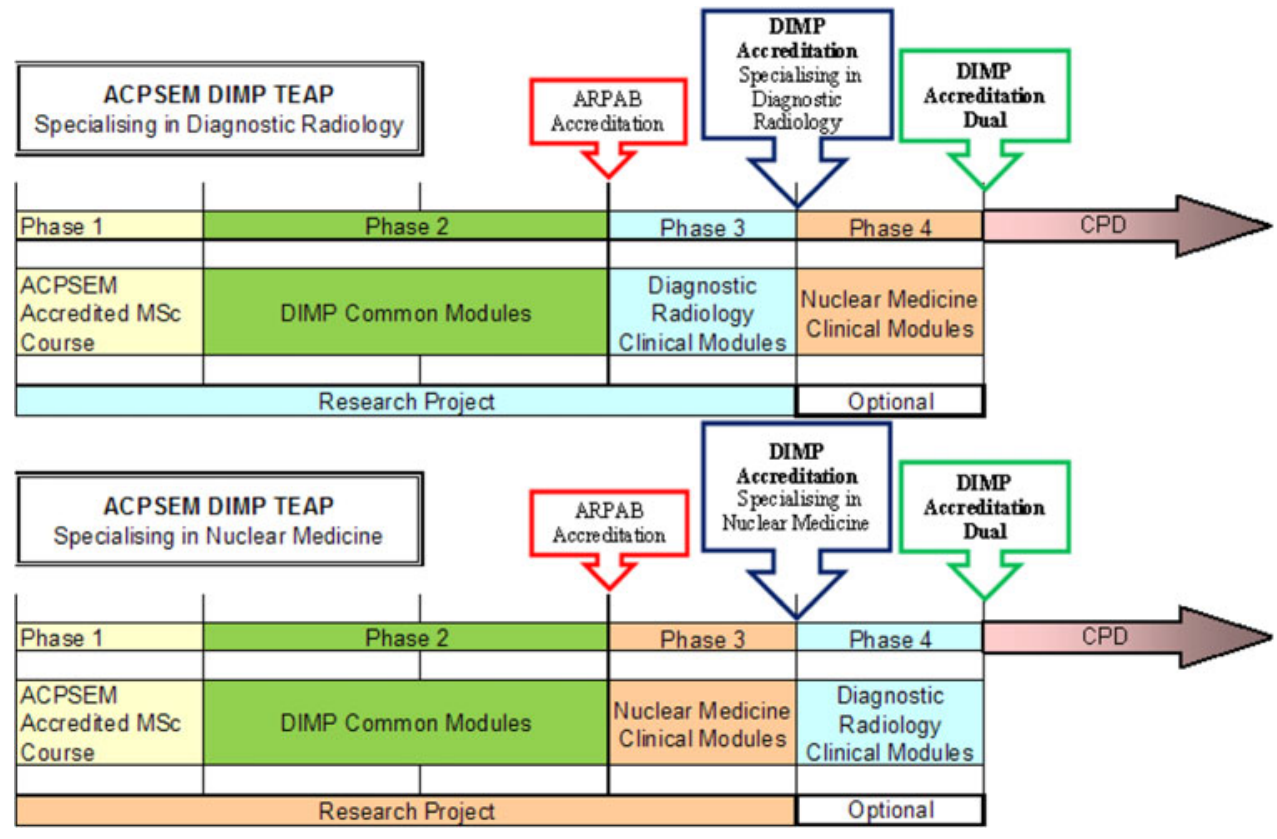


competency in all of the core tasks which are expected to be undertaken in a large medical imaging facility. On completion of this phase, the registrar should also be capable of handling most radiation safety matters and be eligible to apply for ARPAB accreditation as a certified health physicist.

\section{Phase 3 (1 year)}

Successful completion of Phase 2 is a pre-requisite for Phase 3. In this phase more advanced training modules relating to the DIMP specialty that the registrar has chosen are undertaken. On completion of this phase, the registrar will be eligible to apply for ACPSEM accreditation in diagnostic imaging medical physics, specializing in his/her chosen area. Successful accreditation following this phase will signal the completion of mandatory training from an industrial point of view.

\section{Phase 4 (1 year: optional)}

This is an optional post-accreditation phase of training in which the registrar undertakes the Phase 3 modules of an additional DIMP specialty. On completion of this phase, the registrar will be eligible to apply for ACPSEM dual accreditation in both diagnostic imaging medical specialties. It is possible that modules in this phase could be spread over a longer time period.

DIMP registrars will cover the same material in Phases 1 and 2. In Phase 2, in particular, common modules relating to both diagnostic radiology and nuclear medicine will be covered (this is achieved through the identification of common elements and "harmonizing" of some of the basic modules from both specialties). In Phase 3, depending on the needs of the department and registrar, streaming takes place into either the diagnostic radiology or nuclear medicine specialty. Modules in Phases 2 and 3 can be taken in any order, or concurrently (with the proviso that any prerequisites are met) depending on the requirements of the registrar and his/her employer. Registrars may also be required to undertake a research project in their particular specialty which will start during Phase 1 and be completed by the end of Phase 3 . The timeline here simply reflects the relative amount of time allocated to each phase; the overall time taken for accreditation (beginning of phase 1 to the end of Phase 3 will typically be about 4 years. Phase 4 (optional), which leads to specialization in both modalities, will require the completion of some additional modules.

Training modules

32 diagnostic radiology (DR), 44 nuclear medicine (NM) and 15 common (C) modules have been developed. These are based on IAEA training modules, many of which were developed in conjunction with ACPSEM members, and have been reviewed by ACPSEM Accreditation Panels, the DIMP Working Group and other interested parties. The common modules will be mandatory for both the nuclear medicine and diagnostic radiology specialties; the selection of DR and NM modules required will depend on the chosen speciality These modules can be undertaken in any order (with the proviso that any pre-requisites are met) and more than one module can be undertaken at a time.

\section{DIMP module handler}

In order to simplify navigation through the modules, and to facilitate ongoing editing, a Module Handler has been written using Microsoft EXCEL. This has been designed as a dynamic instrument to facilitate the editing, organization and maintenance of training modules in a flexible manner as controlled documents. It also allows both supervisors and registrars to track/record progress and maintain a portfolio folder for each phase of training. It is hoped that this tool will ease the administrative burden on supervisors and ensure a uniform method of recording progress. A typical screen from the handler is shown in Fig. 4.

\section{Clinical training facilities}

Although the process of accrediting training facilities will be similar for both ROMPs and DIMPs, there are problems associated with the current paucity of experienced DIMPs that will need to be addressed. Individual departmental accreditation may not be possible in many cases. For this reason, it may be necessary to accredit training facilities on a regional basis; a regional facility (or cell) will need to meet the following criteria:

- The region must be geographically small enough for registrars to easily commute between the various centres making up the region.

- There must be sufficient experienced DIMPs in the region to supervise (with the aid of preceptors) the training of the number of registrars in the region. Based on other programmes, the experienced DIMP:registrar ratio is aimed to be $1: 1$.

- There should be sufficient test equipment, phantoms and other resources within the region that can be used for registrars in their training.

- The region must have a range of medical imaging and ancillary equipment, in the areas of CT, MRI, nuclear medicine and ultrasound.

- The region must offer a range of medical imaging services which employ medical practitioners trained in nuclear medicine and diagnostic radiology. 


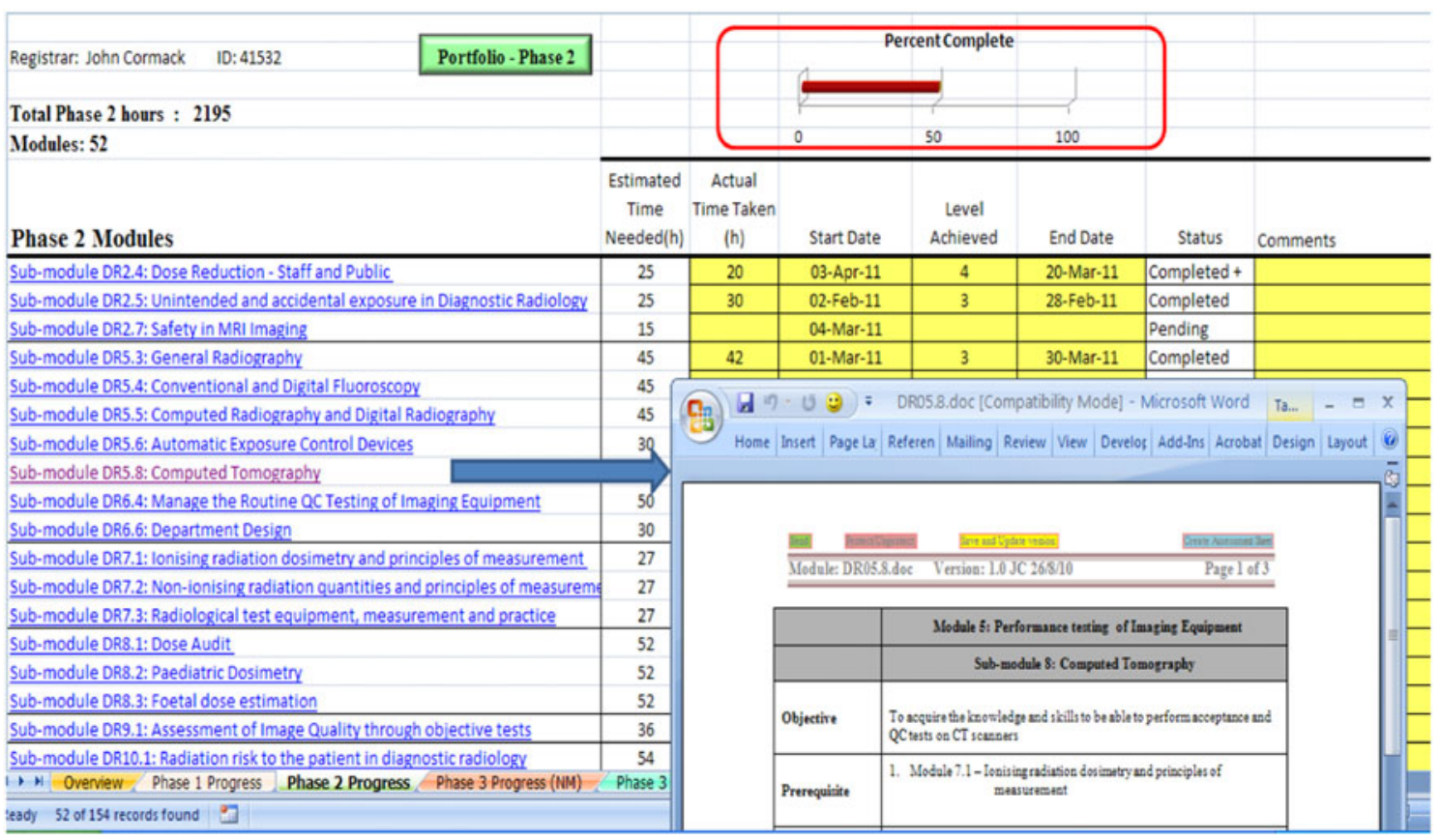

Fig. 4 Typical screen from the DIMP module handler showing a linked module and progress statistics

- Books and other documents associated with the training programme should be readily available for use by DIMP registrars.

\section{Arrangements for registrars currently embarked on existing training programmes}

Existing training programmes for diagnostic radiology and nuclear medicine are currently operational and several registrars have embarked upon them. Mechanisms will be put in place to ensure that these registrars can successfully complete these programmes, and that they are not disadvantaged in any way by the introduction of an updated programme.

Some of the training modules from the new TEAP may also be useful to registrars engaged with existing programmes, and they will be encouraged to use them if they so wish. In particular, all current registrars will be encouraged undertake Module C11 (Radiation shielding considerations in design of new facilities) as this may be crucial for certification in shielding design and verification.

\section{Workforce issues}

Workforce analysis and sustainability

A results of a workforce analysis based on a 4 year training programme is shown below in Fig. 5. This analysis is based on the induction of two staggered groups of 20 registrars, one in 2011, and one in 2012. It has been assumed that it takes 4 years for the registrar to be fully trained and have a full impact (i.e., the registrar has very little impact until after his/her first year of clinical training and becomes progressively more useful each year). A group of 20 registrars will therefore take 4 years to become equivalent to 20 fully trained physicists.

An intake of four experienced physicists per annum over a period of 6 years (compensating for the projected attrition rate) has also been assumed. In addition, a steady intake of at least four registrars per annum (in Australia and New Zealand) from 2014 will also be required to maintain the status quo.

Allowing for attrition, this strategy will bridge the current gap between actual and required numbers by 2016 and maintain a steady workforce numbers thereafter.

It should be noted that no provision has been made here for any increases in demand which may accrue over time this may have to be assessed and factored in at a later stage by increasing the annual registrar intake.

It should be noted that this strategy will not be effective unless permanent positions which extend beyond the training period are made available for all registrars.

\section{What still needs to be done}

Drafting of the new TEAP (see above) is now complete. This now needs to be ratified by the Diagnostic Radiology 
Fig. 5 Effective DIMP workforce trends with and without intervention

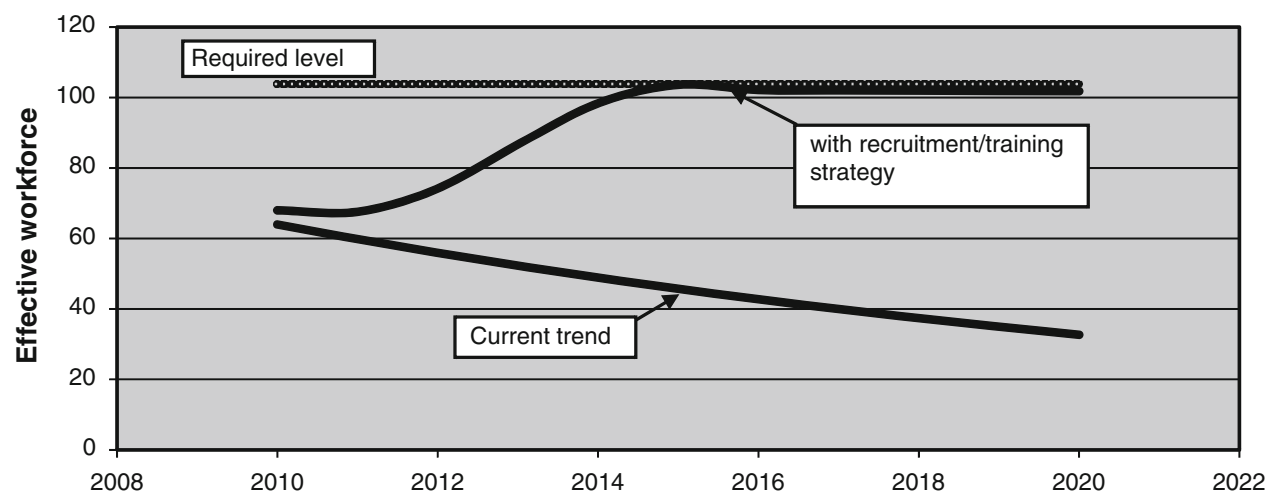

and Nuclear Medicine Panels and the ACPSEM Professional Standards Board. Nothing is yet cast in stone, and it is fully expected that rationalisation and modification of the programme is likely to be an ongoing process as it is implemented.

Although the structure of the new DIMP TEAP has been established, it will be the mechanics (and politics!) of implementation, funding and encouraging participation that are likely to present the biggest problems. The nature of DIMP training and the paucity of experienced DIMPS may necessitate a model that is different from that used for radiation oncology medical physicists. Some creative thinking may be required here.

The generous support of the Department of Health and Aging (DoHA) is acknowledged in the development of this programme. Additional support at both Federal and State level will be required, however, if this programme is to be properly implemented to address the workforce issues discussed above. The ACPSEM is actively pursuing the support that will be required in the "boot-up" stage of this project (there are some hopeful signs here), and is also looking at a system that will be sustainable in terms of human resources and funding in the long term.

Stay tuned!

\section{References}

1. Howell Round W (2010) A 2009 survey of the Australasian clinical medical physics and biomedical engineering workforce. Australas Phys Eng Sci Med 33:153-162

2. NCRP Report No. 160 (2009) Ionizing radiation exposure of the population of the United States. National council on radiation protection and measurements, Bethesda

3. Fazel et al (2009) Exposure to low dose ionizing radiation from medical imaging procedures. N Engl J Med 361(9):849-857

4. UNSCEAR Report (2008) Sources and effects of ionizing radiation: health effects due to radiation from the Chernobyl accident, vol 2, Annex D. United Nations, New York

5. Guidelines for the Provision of Physics Support to Nuclear Medicine (1999) Nucl Med Commun 20:781-787

6. Criteria for the staffing levels in a Physics Department (1997) The European Federation of Organisations for medical Physics (EU)

7. Staffing levels and responsibilities of physicists in diagnostic radiology (1991) AAPM Report Number 33AAPM Report No. 33. American Institute of Physics 